\title{
ANÁLISIS DEL DISEÑO DE INSTRUMENTOS DE EVALUACIÓN IMPLEMENTADOS DESDE DOS CONTEXTOS CURRICULARES
}

\author{
Susana González de Galindo \\ Profesora de la Universidad Nacional \\ de Tucumán. Argentina \\ Leonor Colombo de Cudmani \\ Profesora Emérita de la Universidad Nacional \\ de Tucumán. Argentina
}

\author{
Recibido 8-III-2004 • Aceptado 11-VI-2004
}

\begin{abstract}
Resumen: Esta investigación forma parte de una más abarcativa sobre la problemática de resignificar la clase magistral del Cálculo para cursos superpoblados de primer año, de una Facultad de Ciencias, recurriendo en las clases al uso de una guía elaborada según principios sostenidos por Teorías cognitivas del aprendizaje sostenidas por Piaget, Ausubel, Vigotsky, entre otros.

En este trabajo se investigó si existieron diferencias significativas en el diseño de los exámenes finales que rindieron los alumnos que habían cursado la asignatura en 2001 (año en el que se implementó la nueva metodología) y en 1999 (enseñanza tradicional). Se analizaron los tipos de actividades según los criterios derivados del marco teórico y de los Estándares de Evaluación para la Educación Matemática (NCTM, 1989).

Se concluyó que en la enseñanza tradicional de 1999, las actividades de los exámenes pretendían verificar si los conocimientos transmitidos habían sido asimilados. Las de 2001, por el contrario, satisficieron en buena medida, las pautas establecidas en los Estándares de evaluación seleccionados, lo que significa un importante avance en el proceso de evaluación que se venía implementando.

Esta conclusión ratificaría las conclusiones obtenidas del análisis de otros instrumentos diseñados para evaluar la nueva estrategia metodológica estimulando a implementarla en otros contenidos.
\end{abstract}

\section{Introducción}

Matemática I es una de las asignaturas del primer cuatrimestre de primer año, del currículo de las carreras que se cursan en la Facultad de Bioquímica, Química y Farmacia de la Universidad Nacional de Tucumán, Argentina. En ella se desarrollan los conceptos básicos del Cálculo Diferencial e Integral de una variable.

El presente informe forma parte de una investigación más abarcativa sobre la problemática de resignificar, en esta asignatura, la clase magistral para aulas multitudinarias, dentro de un nuevo modelo de aprendizaje (González de Galindo et al., 2000; González de Galindo y Villalonga de García, 2001; González de Galindo y Colombo de Cudmani, 2001).

En el sistema de transmisión-recepción, característico de las clases teóricas implementadas en esta asignatura hasta 1999, una de las deficiencias más notables era la escasa actividad del alumno, reducida 
Palabras clave: Aulas Multitudinarias, Aprendizaje Significativo, Material Didáctico, Evaluación, Diseño de los Instrumentos.

\begin{abstract}
This research study is part of a more extensive work about the problem of giving new significance to the traditional class of Calculus directed to overcrowded courses of first course in a School of Science. The course is taught using a guide.

The idea was to study if there were significant differences in the design of final exams taken by students who had attended the subject in 2001 (when the new methodology was introduced) and the one taken by those who had attended the course in 1999 (traditional teaching). The types of activities were analyzed according to the criteria derived from the theoretical frame and the Standards of Evaluation for the Teaching of Mathematics (NCTM, 1989).The conclusion was that in the 1999 traditional teaching, the activities of the exams aimed to verify if the given acknowledgment had been assimilated. On the contrary, the 2001 activities extensively satisfied the rules established on the Standards of the selected evaluation. This means that there was an important advance in the process of evaluation that had been used.This conclusion confirms the ones obtained from the analysis of other instruments designed to evaluate the new methodological strategy. Teachers are encouraged to introduce this strategy in other contents.
\end{abstract}

Key words: Croweded Classes, Significant Learning, Didactic Material, Evaluation, Design of Instruments. prácticamente a la toma de apuntes. Los estudiantes se mostraban poco motivados y existía escasa comunicación. Los algoritmos estereotipados y casi automáticos suplían, en general, a los razonamientos característicos de la Matemática. En las clases teóricas y en las de resolución de problemas, no se abrían espacios a otros tipos de actividades. Sin embargo, ante la imposibilidad institucional de implementar propuestas integrales superadoras, se decidió avanzar, por lo menos, en lo que se refiere a la clase magistral.

Basándose en los principios sostenidos por distintas tendencias pedagógicas contemporáneas de base cognitiva, en 2001, se implementó en las clases teóricas, una estrategia didáctica que consistió en el uso de una guía teórico práctica. Ésta fue elaborada desde una óptica constructivista, y se caracterizó por presentar "huecos", a ser completados por los alumnos tras un proceso de elaboración grupal e individual. La necesidad de llenar estos espacios permitió generar actividades de diversos tipos, para desarrollar competencias fundamentales para lograr aprendizajes significativos. Factores externos imposibilitaron la implementación de un grupo experimental y otro de control. Se llevó a cabo un estudio observacional, durante el período estipulado, dentro del cronograma de toda la asignatura, para el aprendizaje de los temas desarrollados en la misma. Correspondería a lo que se ha dado en llamar investigación en la acción con la metodología de la observación participante. Los alumnos que participaron fueron 196.

Durante el desarrollo de las clases se consideró conveniente limitar la información a aquellos conocimientos que el estudiante no pudiera lograr por sí mismo, complementándolas con una guía de actividades diseñada de modo que se estimularan los cuestionamientos, la formulación de hipótesis, la conexión entre contenidos 
y el cambio de representaciones (Arcavi, 1999). Así, el énfasis en el aula estuvo puesto en la participación del alumno, para lograr que sea el estudiante el que generara y construyera comprensión.

Para elaborar el material y validarlo con datos experimentales, se elaboró un prototipo sobre el tema: Función creciente y decreciente, extremos relativos, concavidad, puntos de inflexión y graficación aproximada de funciones.

En este estudio se consideró como variable independiente al uso de la guía teórico práctica y como dependiente a la calidad de la asimilación de los conocimientos, referida tanto al grado de corrección de la acción, como a las dos cualidades siguientes de la acción: grado de reflexión y grado de generalización.

La definición conceptual de cada una de estas dimensiones es la siguiente:

Grado de corrección: grado en el que el conocimiento procesual del alumno se realiza conforme a las conceptuaciones científicas.
Grado de reflexión: grado de la capacidad del alumno para razonar matemáticamente.

Grado de generalización: grado de la capacidad del alumno para resolver distintas situaciones problemáticas, al aplicar los conocimientos matemáticos.

La definición operacional de cada una de estas dimensiones, estuvo constituida por el puntaje asignado al ítem diseñado para evaluar esa dimensión (González de Galindo y Colombo de Cudmani, 2002).

Desde una óptica constructivista, el objetivo de producir aprendizajes significativos de los conocimientos tiene consecuencias que afectan a los distintos aspectos del proceso de enseñanza aprendizaje: concepción del currículo, estructuración del trabajo en el aula, modalidades de realización de los trabajos prácticos, abordajes de problemas, introducción de conceptos, evaluación, entre otros. Por ello, para evaluar la experiencia en su totalidad, la recolección de datos se hizo recurriendo a distintas fuentes y procedimientos:

\begin{tabular}{|l|l|}
\hline FUENTES & PROCEDIMIENTOS \\
\hline Actividades en Clase & $*$ Observación sistemática en el aula. \\
\hline Estudiantes & $\begin{array}{l}* \text { Encuesta } \\
* \text { Exámenes parciales. * Exámenes finales. }\end{array}$ \\
\hline Profesores & $\begin{array}{l}* \text { Sesiones para la validación del material didáctico. } \\
* \text { Observaciones de las clases. } \quad * \text { Encuesta. }\end{array}$ \\
\hline
\end{tabular}

En una investigación anterior, se analizó la información suministrada por el examen correspondiente al segundo parcial de esta asignatura. Los resultados evidenciarían una mejora en el grado de corrección de la acción, cuando se recurre al uso, en las clases multitudinarias, de un material didáctico con las características ya especificadas. Pudo apreciarse, además, que en el examen de 2001, se incluyeron actividades 
destinadas a evaluar específicamente el grado de reflexión y el de aplicación, lo que representa un progreso con respecto al tipo de tareas propuestas, hasta ese momento, en los exámenes parciales. En estas dimensiones, un importante número de alumnos tuvo un desempeño satisfactorio (González de Galindo y Colombo de Cudmani, 2001).

Posteriormente se analizó la encuesta realizada a los alumnos, a fin de comprobar si la experiencia se había ajustado a los lineamientos propios de un aprendizaje constructivista según lo entienden Piaget, Ausubel, y Vigotsky, entre otros (Coll y Martí, 1994; Moreira, 1997, González de Galindo y Colombo de Cudmani, 2002). Las conclusiones de esa encuesta indicarían que los objetivos a alcanzar con la nueva estrategia, se lograron en gran medida, y estimulan a implementar la nueva estrategia en todos los temas de esta asignatura.

$\mathrm{Al}$ abordar la problemática de la evaluación, la que debiera estar integrada al modelo de enseñanza aprendizaje que se haya seleccionado, y en la cual debieran apreciarse cambios sustanciales respecto a la práctica evaluadora tradicional, al haberse introducido modificaciones en uno de los aspectos didácticos del proceso, se consideró necesario investigar, en primer lugar, si existían diferencias significativas en el diseño de los instrumentos empleados para evaluar los aprendizajes de los alumnos que habían trabajado con la nueva metodología, y el de aquellos empleados para evaluar a quienes habían recibido instrucción tradicional. Estos instrumentos fueron los exámenes finales que rindieron los alumnos que habían cursado la asignatura en los años 2001 (año en el que se implementó la nueva metodología) y 1999 (año con enseñanza tradicional). En otras palabras, se pretende examinar si una enseñanza que responde a enfoques alternativos plantea necesarias modificaciones en la forma de evaluar, comparándola con la práctica evaluadora habitual. Este es precisamente el objetivo de este trabajo.

\section{Hipótesis}

Las hipótesis que guiaron el estudio comparativo de ambos tipos de exámenes fueron:

a) La evaluación usual en la cátedra no favorecía aprendizajes significativos, en el sentido que las actividades evaluativas contribuyeran a integrar los conocimientos en forma no arbitraria dentro de las estructuras conceptuales de los alumnos (Ausubel, 1978, Villalonga de García y Colombo de Cudmani, 2002).

b) La nueva estrategia didáctica implementada en las clases implica una concepción y práctica de la evaluación distinta de la tradicional, y se refleja en el contenido de los exámenes.

Las conclusiones a las que se arriben en este estudio se contrastarán con estas hipótesis y serán trianguladas con las obtenidas en los estudios anteriormente citados.

\section{Marco Téorico}

La fundamentación teórica se logró complementando los aportes de las siguientes teorías: Psicogenética de Piaget, del Aprendizaje Significativo de Ausubel y del Enfoque Histórico Cultural de Vigotsky con la Teoría de la actividad de Leontiev (Czar y Pizarro de Raya, 1993; Coll y Martí, 1994; Moreira, 1997). Los lineamientos teóricos y las pautas a tener en cuenta en la concepción de la construcción del conocimiento, fueron explicitados en un trabajo anterior (González de Galindo y Colombo de Cudmani, 2001).

En un trabajo previo se expusieron las características de las prácticas evaluativas realizadas en esta asignatura, llegando a la conclusión que las mismas están principalmente relacionadas con los procesos de 
medición de los aprendizajes puramente conceptuales $\mathrm{y}$, en general, de repetición memorística verbal y algorítmica, y en una medida significativamente menor, con el análisis de las dificultades en la adquisición, comprensión o transferencia de conocimientos. Predomina la idea de acreditación, aún cuando según Díaz Barriga las lógicas de la evaluación y de la acreditación son sustancialmente distintas: la de la acreditación es de carácter institucional, mientras que la lógica de la evaluación es la de comprender el proceso educativo (Díaz Barriga, 1990; González de Galindo y Nieva de del Pino, 2002).

En este trabajo se realizará el estudio comparativo del contenido de los exámenes, ya que el contenido de un examen pone de manifiesto los aspectos a los que se concede más importancia. Dado que el objetivo que se persigue es analizar si el diseño de los instrumentos empleados para evaluar a los alumnos que habían completado el cursado de esta asignatura en 2001, difiere de los instrumentos empleados en 1999, año con enseñanza tradicional, resulta necesario analizar, en particular, la evaluación desde el marco teórico elegido. $\mathrm{O}$ sea resulta necesario analizar el papel y las características que debiera tener la evaluación para ser coherente con los fundamentos teóricos seleccionados.

La evaluación desde una nueva perspectiva: $\mathrm{El}$ marco teórico establecido lleva a considerar a la evaluación como el proceso a través del cual se obtiene, analiza e interpreta información, para valorar alternativas y argumentar decisiones que revitalicen, cualitativamente, los procesos de enseñanza aprendizaje (Villagra de Burgos, 1993). Esta perspectiva tiene las siguientes implicaciones:

\section{La evaluación}

a) es una parte del proceso didáctico, y no un acto final desprendido de las acciones propias de la enseñanza y del aprendizaje,

b) es un denunciante de todo el proceso,

c) lleva al docente a reconocer la importancia de autoevaluarse, asumiendo su propuesta de enseñanza con carácter experimental. La dinámica de esta revisión debiera realizarse desde un punto de vista personal, revisando sus conductas en prácticas manifiestas, y analizando la profundidad de su conocimiento disciplinario, junto a la postura filosófica, pedagógica y sociológica que constituye su visión del mundo. Pero también, monitoreando su accionar con otros docentes que reúnan condiciones de confidencialidad, confianza y reciprocidad, para obtener información a través de críticas constructivas.

d) rescata la importancia de que los alumnos se auto evalúen. Esta autoevaluación resulta ser una habilidad a construir, y corresponde incluirla como estrategia de enseñanza.

e) requiere que se consideren los aprendizajes con que los alumnos acceden a la nueva temática. Es necesario, entonces, implementar una evaluación inicial o diagnóstica la que permitirá modificar la programación tanto como sea posible, para que haya una mejor adecuación entre capacidad cognitiva y currículo Es importante que esta prueba cumpla sobre todo con el requisito de validez predictiva (Díaz Barriga y Hernández Rojas, 1997; Nunnally, 1970).

f) debería mejorar la comunicación. Según Bruner el docente debe permitir que los estudiantes planteen sus hipótesis más arriesgadas, sus intuiciones o sus interrogantes, sin 
que los mismos teman que con estas intervenciones, se descubran sus ignorancias. De esta manera se estimularía la zona de desarrollo próximo, tal como la denomina Vigotsky. Las preguntas de los alumnos, permiten al docente reconocer las maneras en que aquellos se interrogan respecto de un tema, la naturaleza de los errores o las falsas concepciones (Bruner, 1997; Czar y Pizarro de Raya, 1993).

Según Alonso, Gil y Martínez Torregrosa (1992) las características de este nuevo concepto de evaluación son:

a) Las instancias evaluativas deben ser percibidas por el alumno como oportunidades para recibir ayuda y descubrir sus propios avances, dificultades y necesidades.

b) Si el objetivo es conseguir aprendizajes significativos, al evaluar un determinado conocimiento deben considerarse los contenidos conceptuales, procedimentales y actitudinales involucrados en el mismo, abandonando el hábito de evaluar la capacidad de memorizar conocimientos teóricos y sus respectivas aplicaciones prácticas.

c) Se torna necesaria la implementación de una evaluación permanente, realizada a lo largo de todo el proceso e integrada al mismo, que retroalimente y permita adoptar las medidas superadoras necesarias (Colombo, Pesa y Salinas, 1986).

Para situar el marco teórico en la enseñanza de la Matemática, se analizan las pautas establecidas por los Estándares de Evaluación para la Educación Matemática (NCTM, 1989) respecto a la evaluación y a sus instrumentos.

\section{a) Principios establecidos por los Estándares de Evaluación}

Según los Estándares de Evaluación para la Educación Matemática "el principal objetivo de la evaluación es ayudar al profesor a comprender mejor lo que los estudiantes saben, y a tomar decisiones docentes significativas. La atención se centra en lo que ocurre en el aula con la interacción de profesores y alumnos" (NCTM, 1989: 195). Estos Estándares de Evaluación proponen que:

- la evaluación de los alumnos quede integrada en la docencia,

- se utilicen distintos métodos de evaluación,

se evalúen todos los aspectos del conocimiento matemático y sus conexiones,

se consideren de igual forma la docencia y el currículo a la hora de enjuiciar la calidad de un programa." (NCTM, 1989:196).

En particular, considerando que el interés radica en evaluar la variable calidad de la asimilación, surge la siguiente pregunta: ¿qué establecen los estándares de evaluación respecto a las distintas dimensiones de esta variable? Se consideró que al evaluar cada una de las tres dimensiones debían considerarse las pautas establecidas en el Estándar 8 sobre la evaluación de Conceptos matemáticos:

"La evaluación del conocimiento y las estructuras conceptuales de los alumnos sobre conceptos matemáticos debe ofrecer evidencia de que son capaces de -dar nombre, verbalizar y definir conceptos; -identificar y generar ejemplos válidos y no válidos; -utilizar modelos, diagramas y símbolos para representar conceptos; -pasar de un modo de representación a otro; -reconocer 
los diversos significados e interpretaciones de los conceptos; -identificar propiedades de un concepto determinado y reconocer las condiciones que determinan un concepto en particular; -comparar y contrastar conceptos.

Además, la evaluación debe ofrecer evidencia de hasta qué grado han conectado los alumnos el conocimiento de diversos conceptos." (NCTM, 1989:230).

Además del Estándar 8, referido al conocimiento conceptual y relacionado con las tres dimensiones de la variable, se considerará, al evaluar cada dimensión en particular, el Estándar más estrechamente vinculado a la misma. Se detallan a continuación los Estándares seleccionados para analizar cada dimensión:

- Grado de corrección: Al evaluarlo se tendrán presentes los principios sobre los procedimientos matemáticos establecidos en el Estándar 9:

"La evaluación del conocimiento procesual de los alumnos debe ofrecer evidencia de que son capaces de:

-reconocer cuándo es adecuado un procedimiento; -explicar las razones para los distintos pasos de un procedimiento; -llevar a cabo un procedimiento de forma fiable y eficaz; -verificar el resultado de un procedimiento empíricamente (por ej. utilizando modelos) o analíticamente;- reconocer procedimientos correctos e incorrectos; -generar procedimientos nuevos y ampliar o modificar los ya conocidos, -reconocer la naturaleza y el papel que cumplen los procedimientos dentro de las matemáticas" (NCTM, 1989:235).

- Grado de reflexión: El Estándar 7, referido a la evaluación del Razonamiento, es el seleccionado como referente para evaluar el grado de reflexión. El mismo especifica que:
"La evaluación de la capacidad que tengan los alumnos para razonar matemáticamente debe ofrecer evidencia de que son capaces de:

- utilizar el razonamiento inductivo para reconocer patrones y formular conjeturas; utilizar el razonamiento para desarrollar argumentos plausibles de enunciados matemáticos; utilizar el razonamiento proporcional y espacial para resolver problemas; utilizar el razonamiento deductivo para verificar una conclusión, juzgar la validez de un argumento y construir argumentos válidos; analizar situaciones para hallar propiedades y estructuras comunes; reconocer la naturaleza axiomática de las matemáticas." (NCTM, 1989:226).

- Grado de aplicación: El Estándar 5 establece con respecto a la Resolución de problemas lo siguiente:

"La evaluación de la capacidad que tenga el alumno de utilizar las matemáticas para la resolución de problemas debe mostrar evidencia de que son capaces de-formular problemas; aplicar diversas estrategias para resolver problemas; -resolver problemas;-comprobar e interpretar resultados;-generalizar soluciones." (NCTM, 1989:216).

La consideración de todos estos principios conduce a replantear los procesos evaluativos, incorporándole modificaciones que los transformen en un aspecto coherente con la nueva metodología adoptada.

\section{b) Los instrumentos de evaluación en la nueva perspectiva evaluadora}

Con respecto a los instrumentos de evaluación, los estándares indican que los mismos deben permitir al docente entender la forma que tienen los alumnos 
de percibir ideas y procesos matemáticos y contribuir a identificar áreas concretas que resulten problemáticas, con objeto de mejorar el proceso de enseñanza aprendizaje (NCTM, 1989).

Para lograr que el aprendizaje sea realmente un proceso de cambio conceptual, procedimental y actitudinal, es necesario incluir en las pruebas actividades (preguntas, ejercicios y problemas) de los siguientes tipos:

1) Actividades centradas en el manejo significativo de los conceptos, que no impliquen la simple repetición de conocimientos transmitidos;

2) Actividades que involucren el manejo de distintos aspectos de la metodología científica: planteamientos cualitativos, formulación de hipótesis y consideración de situaciones límites, análisis cualitativos de resultados, etc;

3) Actividades en las que se aprecie la aplicación de los temas desarrollados en clase, a la vida cotidiana, a las ciencias aplicadas o a la tecnología.

Incluir este tipo de actividades en los exámenes indicaría que se pretende influir en el aprendizaje en el momento mismo del examen, ocasión propicia dada la implicación de los alumnos en esta actividad, propiciando situaciones de retroalimentación entre los alumnos y sus propios procesos de aprendizaje.

\section{Metodología}

Para estudiar si existían diferencias entre el diseño de los instrumentos utilizados para evaluar a los alumnos que habían experimentado en 2001 la nueva metodología, y el diseño de los instrumentos de 1999 (enseñanza tradicional), se analizaron los enunciados de los exámenes finales correspondientes, respectivamente, a las primeras diez mesas examinadoras posteriores al cursado de la asignatura. Cada uno de estos exámenes finales pretendía evaluar los aprendizajes logrados por los alumnos en los diversos contenidos de la asignatura. De la totalidad de las actividades propuestas en cada instrumento, sólo se analizaron las relacionadas con el tema desarrollado en la guía. En esta tarea se tuvieron presentes, los criterios que derivan del marco teórico enunciado.

Siguiendo a Taylor y Bogdan (1986) se realizó un análisis comprensivo de los datos, efectuándose: 1) Sucesivas lecturas de los enunciados de los exámenes; 2) determinación de las categorías de análisis; 3) codificación de las tareas; 4) separación de las tareas en las categorías.

Realizado este proceso, fue posible distinguir las siguientes categorías y dimensiones:

1) Actividades que favorecen un aprendizaje repetitivo: a) Ejercicios con énfasis en destrezas operativas, b) Contenidos teóricos que pueden responderse recurriendo a la memoria (definiciones de conceptos, enunciados de teoremas), c) Contenidos teóricos que implican manejo significativo de conceptos, pero que ya fueron desarrollados en clase (demostraciones de teoremas).

2) Actividades que favorecen un aprendizaje significativo: d) Actividades que obligan al alumno a realizar un manejo significativo de conceptos, e) Actividades de aplicación de los contenidos teóricos a las ciencias, técnica y vida diaria, f) Actividades de autorregulación. 


\section{Resultados}

Se presentan en la siguiente tabla los resultados del análisis comparativo de los tipos de actividades de evaluación, relacionadas con el tema de la guía, incluidas en los diez exámenes implementados en una enseñanza tradicional (27 actividades en total) y en una enseñanza fundamentada en principios constructivistas (57 actividades).
El gráfico 1 ilustra, para cada muestra, el porcentaje de los dos tipos de actividades:

Los resultados confirmarían las hipótesis enunciadas, puesto que aproximadamente el $81 \%$ de las actividades propuestas en los exámenes correspondientes a una enseñanza tradicional favorecen aprendizajes repetitivos. Dentro de estas actividades presentan igual porcentaje (30\%) las cuestiones de teoría que requieren sólo de la memoria y aquellas en las

\begin{tabular}{|l|c|c|}
\hline \multirow{2}{*}{ ACTIVIDADES DE EVALUACIÓN } & \multicolumn{2}{|c|}{ TIPOS DE ENSEÑNZA } \\
\cline { 2 - 3 } & $\begin{array}{c}\text { Enseñanza } \\
\text { tradicional }\end{array}$ & $\begin{array}{c}\text { Enseñanza con } \\
\text { fundamentación } \\
\text { constructiva }\end{array}$ \\
\hline a) Ejercicios con énfasis en destrezas operativas. & $21 \%$ & $7 \%$ \\
\hline $\begin{array}{l}\text { b) Contenidos teóricos que pueden responderse } \\
\text { recurriendo a la memoria (definiciones de } \\
\text { conceptos, enunciados de teoremas). }\end{array}$ & $30 \%$ & $21 \%$ \\
\hline $\begin{array}{l}\text { c) Contenidos teóricos que implican manejo } \\
\text { significativo de conceptos, pero que ya fueron } \\
\text { desarrollados en clase (demostraciones de teoremas). }\end{array}$ & $30 \%$ & $\mathbf{3 7 \%}$ \\
\hline $\begin{array}{l}\text { Total de actividades que no fomentan } \\
\text { un aprendizaje significativo }\end{array}$ & $\mathbf{8 1 \%}$ & $\mathbf{3 7 \%}$ \\
\hline $\begin{array}{l}\text { d) Actividades que obligan al alumno a realizar } \\
\text { un manejo significativo de conceptos. }\end{array}$ & $19 \%$ & $\mathbf{6 3 \%}$ \\
\hline $\begin{array}{l}\text { Total de actividades que pueden favorecer } \\
\text { un aprendizaje significativo }\end{array}$ & $0 \%$ & $6 \%$ \\
\hline $\begin{array}{l}\text { e) Actividades de aplicación de los contenidos } \\
\text { teóricos a las Ciencias, Técnica y vida diaria. }\end{array}$ & $\mathbf{1 9 \%}$ & \\
\hline \begin{tabular}{l} 
f) Actividades de autorregulación. \\
\hline
\end{tabular} & & \\
\hline
\end{tabular}


que subyace un manejo significativo de conceptos pero que ya fueron desarrolladas en las clases teóricas (demostraciones de teoremas). Un $21 \%$ de las actividades evalúan destrezas operativas. Sólo el 19\% de las actividades ponen énfasis en la comprensión y manejo significativo de conceptos, y es nulo el porcentaje de actividades relativas a la autorregulación y a la aplicación de contenidos teóricos a las Ciencias y a problemas de la vida diaria.

Por el contrario, en los exámenes implementados luego de una enseñanza basada en principios constructivistas, el porcentaje de actividades que ponen énfasis en el manejo significativo de conceptos alcanza al $63 \%$. En un porcentaje pequeño, figuran las destinadas a autorregulación y a aplicaciones de los contenidos teóricos.

Cabe destacar que hay objetivos de aprendizaje que no son detectados eficientemente con el instrumento analizado en este trabajo. Ellos requieren de otras modalidades de evaluación como observación sistemática, entrevistas, ejecución de proyectos integradores, entre otros.
Análisis de los tipos de actividades incluidas en los instrumentos, de acuerdo a los criterios establecidos en los Estándares de evaluación

Exámenes de 1999: Las actividades incluidas en estos exámenes, fueron coherentes con el modelo de enseñanza por transmisión de contenidos elaborados. En cuanto a la evaluación de conceptos, favorecían aprendizajes repetitivos porque podían realizarse, en su mayoría, mediante una aplicación dirigida de los contenidos, o recurriendo a la repetición memorística. Consistían en definiciones de conceptos, enumeración de propiedades y enunciados y demostraciones de teoremas desarrollados por el docente en las clases teóricas. En todos los instrumentos, este tipo de actividades tenía una representación superior al 70\% de la totalidad de tareas propuestas. Por otra parte, en ningún examen se solicitó la formulación y análisis de ejemplos válidos o no válidos, como tampoco el reconocimiento de los distintos significados e 
interpretaciones de un concepto, ni la conexión entre ellos.

Con respecto a cada una de las dimensiones de la variable calidad de asimilación de los conocimientos se puede afirmar lo siguiente:

- Grado de corrección: En cuanto a la evaluación de procedimientos las actividades propuestas servían para constatar el grado de adiestramiento de los alumnos en una destreza operativa, que podía realizarse con un grado limitado de reflexión. Algunas consistían en ejercicios de aplicación directa de la tesis de algún teorema, muchas veces planteadas a continuación de haberse solicitado el enunciado y demostración del mismo. Es decir, no se requerían procesos de selección de estrategias. Se considera que las actividades propuestas satisfacen algunos de los principios establecidos por el Estándar 9: -reconocer cuándo es adecuado un procedimiento (por ejemplo, cuando se requiere analizar extremos relativos a través del Criterio de la segunda derivada), -explicar las razones para los distintos pasos de un procedimiento,-llevar a cabo un procedimiento de forma fiable y eficaz.

Sin embargo, hay ausencia de actividades con énfasis en la verificación de los resultados obtenidos, generación de procedimientos nuevos y aplicación o modificación de los ya conocidos.

- Grado de reflexión: En la evaluación de razonamientos se observó un porcentaje muy bajo de actividades con énfasis en la comprensión y manejo significativo de contenidos conceptuales. Estos exámenes no incluyen actividades en las que sea necesario utilizar el razonamiento para desarrollar argumentos plausibles de enunciados matemáticos, juzgar la validez de un argumento, construir argumentos válidos o analizar situaciones para descubrir propiedades y estructuras comunes.

- Grado de aplicación: No se pudo evaluar la capacidad para resolver problemas al comprobarse ausencia de tales actividades.

Exámenes de 2001: Al tratarse de exámenes finales que pretendían evaluar las competencias logradas por los alumnos en los diversos temas que integran el currículo completo de esta asignatura, sólo se pudieron incluir tres ítemes relativos a los contenidos desarrollados en la guía. Cada uno de los tres ítemes fue diseñado para analizar el logro alcanzado en una de las tres dimensiones de la variable. El análisis de cada ítem se hizo de acuerdo a los principios establecidos en el Estándar 8 (conceptos matemáticos) y en el Estándar, de los tres mencionados anteriormente, que estuviera más estrechamente vinculado a él.

$\mathrm{Al}$ analizar estos exámenes se aprecia una disminución significativa del porcentaje de actividades meramente repetitivas, habiéndose incorporado aspectos que enriquecen el perfil de las pruebas. $\mathrm{Al}$ incluirse tareas con énfasis en el manejo significativo de conceptos, el tipo de procesos cognitivos que se evaluó fue totalmente distinto al tradicional. Por otra parte, no sólo en la guía empleada en las clases, sino también en los exámenes, fueron incorporados aspectos de la relación entre la ciencia, la tecnología y sus implicaciones sociales, para generar en los alumnos una imagen correcta de las ciencias y favorecer así, una mejor actitud hacia el aprendizaje de esta disciplina (Gil, Carrascosa, Furió y Martínez Torregrosa, 1991).

En coherencia con los planteos constructivistas, estos exámenes incluyen situaciones de autorregulación, tales como aquellas en las que se solicita a los alumnos que 
corrijan o critiquen enunciados que contienen algunos de los errores en los que incurren regularmente los alumnos. Por ejemplo, una de las actividades consistía en analizar la validez de las siguientes afirmaciones, solicitándose argumentos que respaldaran las conclusiones:

- $\quad$ Si $f^{\prime}(c)=0$ entonces $\mathrm{f}$ (c) es extremo relativo.

- $\quad$ Si f (c) es extremo relativo, entonces $f^{\prime}(c)=0$.

Otros ítemes que pretendían evaluar tanto el conocimiento sobre conceptos matemáticos, como la capacidad del alumno para razonar matemáticamente, ponían en evidencia si el alumno era capaz de utilizar el razonamiento deductivo para obtener una conclusión, juzgar la validez de un argumento y construir argumentos válidos, realizar conversiones de distintos sistemas de representación semiótica: gráfica, simbólica y coloquial; reconocer los diversos significados e interpretaciones de los conceptos, identificar propiedades de un concepto determinado y reconocer las condiciones que determinan un concepto en particular. Por ejemplo, para desentrañar los conceptos claves implicados en el estudio de una función, una serie de sentencias expresadas en forma algebraica, debían ser convertidas al lenguaje verbal y luego interpretadas gráficamente. Se consideraba que si el alumno no había adquirido tales conceptos en forma significativa, no podría lograr el gráfico que satisfacía las condiciones establecidas.

Es conveniente aclarar que en la distinción de los distintos tipos de actividades realizadas, varias de las que fueron encasilladas como del tipo d) y e), pueden ser vistas como actividades de autorregulación, ya que llevan al alumno a reflexionar y darse cuenta de sus propias carencias o dificultades, en el mismo momento de realizar el examen.
Con respecto a cada una de las dimensiones de la variable calidad de asimilación de los conocimientos, al analizar estos exámenes se puede afirmar lo siguiente:

Grado de corrección: Para establecer comparaciones con los resultados en los aprendizajes obtenidos en 1999, se incluyó, en 2001, en todos los instrumentos, una actividad similar a la incluida en ese año.

Grado de reflexión: Estos exámenes incluyen actividades que pretenden evaluar diversas capacidades, entre ellas: -discriminar entre atributos relevantes e irrelevantes de un concepto cuando el propósito es proporcionar ejemplos válidos y no válidos: -Representar conceptos de distinta manera, reconociendo sus diversos significados. -Aplicar la información que se posee de un determinado concepto a situaciones nuevas.

-Realizar razonamientos tanto intuitivos como formales: razonamiento inductivo, deductivo, así como el desarrollo de argumentos plausibles (aquellos que evidencian sentido común y son matemáticamente correctos). -Reflexionar sobre elementos geométricos y gráficos, para realizar trazos en un sistema de coordenadas cartesianas rectangulares.

Se presentan a continuación ejemplos de las actividades incluidas en los exámenes de 2001, cuyos objetivos específicos eran:

- $\quad$ Identificar y generar ejemplos válidos y no válidos de conceptos: "Grafica una función $f$ continua, que en un punto c de su dominio, verifica las condiciones: $f^{\prime}(c)=0$ y $f(c)$ no es un extremo relativo".

- Identificar propiedades de un determinado concepto: “¿Qué puedes decir 
de la primera derivada en un punto en el que la función tiene extremo relativo?"

- Comparar y contrastar conceptos: “PPuede un punto $P(c, f(c))$ de la gráfica de una función, ser al mismo tiempo máximo relativo y punto de inflexión? Explica y grafica”.

- Integrar el conocimiento conceptual: "Si se sabe que una función f tiene en un punto $c_{1}$ de su dominio un máximo relativo: a) ¿qqué puedes afirmar del crecimiento de la función en los puntos del entorno de $c_{1}$ ?, b) ¿qué puedes decir de la derivada de la función en el punto $c_{1}$ ? c) Si se sabe que la función tiene un mínimo relativo en otro punto $c_{2}$ del dominio ¿es siempre $f\left(c_{1}\right)$ mayor que $f\left(c_{2}\right)$ ?"

- Pasar de un modo de representación a otro: "Grafica una función que cumpla simultáneamente con las siguientes condiciones (expresa en los puntos suspensivos el significado de cada una de esas condiciones):

$f^{\prime}(x)<0$ si $x>2$

$P(2,3)$ es un punto de inflexión de la gráfica de $f$.

$f^{\prime \prime}(x)>0$ si $x>2$ ".

Grado de aplicación: Se entiende por aplicación a aquellos comportamientos que muestran la capacidad y habilidad adquiridas para encarar situaciones particulares concretas, seleccionando y usando correctamente las abstracciones correspondientes. Pero, para que la aplicación sea auténtica, estas situaciones deben ser nuevas, en el sentido que se presentan por primera vez al estudiante (Cudmani, Lozano y Lewin, 1981:
272). Desde este punto de vista, la conducta aplicar implica haber adquirido las capacidades y habilidades que le preceden en la clasificación taxonómica: conocer y comprender. Así, quien sólo conoce y comprende es capaz de usar correctamente una abstracción determinada cuando se le solicite explícitamente. En el nivel de aplicación, por el contrario, el sujeto recurre a la abstracción correcta para encarar el problema, sin necesidad de tales indicaciones expresas.

Según Hernández Fernández (2001) la solución de problemas debe ser un objetivo fundamental. No se trata de aprender definiciones, enunciados y demostraciones de teoremas y procedimientos que permitan resolver determinados tipos de ejercicios. Se debe tratar, por el contrario, de desarrollar estrategias generales que permitan al estudiante buscar bases de orientación adecuadas para resolver los problemas que se le presentan. Pero el Estándar 5 de Evaluación sostiene, que la capacidad de resolver problemas "se va desarrollando con el tiempo como resultado de haber recibido una amplia docencia, haber tenido la oportunidad de resolver problemas de muy distintos tipos y haberse enfrentado a situaciones del mundo real. El avance de los estudiantes debe evaluarse sistemáticamente, deliberada y continuamente, para que se pueda ejercer una influencia efectiva sobre la confianza de los estudiantes y su capacidad para resolver problemas en contextos diversos" (NCTM, 1989:216). Por su parte, Delgado Rubí sostiene que "la enseñanza de la resolución de problemas es una tarea pedagógica difícil, pues deben tenerse en cuenta la formación de múltiples aspectos que conllevan a que el sujeto sea eficiente resolviendo problemas (dentro de una esfera dada del conocimiento) y ésta produce un aprendizaje significativo si el sujeto se enfrenta a situaciones para las cuales no conoce la vía de cómo resolverla" 
(Delgado Rubí, 2001:89). Estas aseveraciones, y el reducido tiempo en el que se desarrolló la experiencia (16 horas), determinaron que, al evaluar la capacidad de resolver problemas, se consideraran sólo las capacidades de: aplicar estrategias y técnicas de resolución de problemas, resolver e interpretar resultados.

Cada uno de los instrumentos del 2001, incluyó un ítem en el que se evaluaron esas capacidades. Las capacidades de formular problemas, seleccionar datos relevantes, detectar datos faltantes y generalizar soluciones, no pudieron ser desarrolladas durante la experiencia, ni consideradas, lógicamente, a la hora de diseñar los instrumentos.

Si bien se reconoce que para evaluar la capacidad para resolver problemas es necesario no sólo analizar exámenes, sino recurrir a distintos métodos (observación del alumno al resolver problemas en forma individual, en pequeños grupos o en discusiones del grupo total de la clase, escuchar a los alumnos discutir sus procesos de resolución de problemas), el elevado número de alumnos impidió apelar a estas metodologías.

\section{Discusión de los resultados}

Se aprecia una diferencia importante en el diseño de los instrumentos correspondientes a los años 1999 y 2001.

Los exámenes correspondientes a 1999 no fueron diseñados para evaluar aprendizajes significativos. Estuvieron destinados, más bien, a verificar si los conocimientos transmitidos habían sido asimilados por los alumnos. Los de 2001, satisfacen en buena medida, las pautas establecidas en los cuatro Estándares de evaluación seleccionados, lo que significa un importante avance en el proceso de evaluación que se venía implementando en esta asignatura, haciéndolo más coherente con una enseñanza orientada por los modelos alternativos.
El tipo de tareas planteadas en estos exámenes llevó al alumno a pensar, "favoreciendo el cambio metodológico de un modo de actuar puramente operativista, a una forma de abordar las situaciones que utilice aspectos de la metodología científica" (Alonso, Gil y Martínez Torregrosa, 1992:135). La tónica estuvo puesta, no tanto en lo procedimental como ocurrió en 1999, sino en comprobar si los alumnos habían logrado captar la esencia de cada concepto, establecer relaciones entre ellos, analizar propiedades y estructuras comunes, diferenciar unos de otros, brindar argumentos para juzgar la validez de una sentencia matemática y aplicar los conocimientos adquiridos para resolver problemas matemáticos vinculados con diferentes áreas del conocimiento y con situaciones de la vida cotidiana.

Algunas de las carencias de la nueva propuesta evaluativa que deberían ser incorporadas gradualmente son:

1. Aún no se han incluido actividades en las que se aprecie si el alumno es capaz de reconocer procedimientos correctos de aquellos incorrectos.

2. Para incentivar la reflexión sobre errores conceptuales, favoreciendo la autorregulación, sería conveniente proponer a los alumnos que analicen gráficas que supuestamente satisfacen condiciones establecidas explícitamente, pero que resultan erróneas, pidiéndoles que brinden argumentos para descalificarlas y que propongan la gráfica correcta. La idea principal radica en presentarles los errores que el docente detecta al corregir pruebas estándar con ejercicios típicos. Tener que corregir respuestas que se saben erróneas, estimula la reflexión sobre los errores cometidos y brinda una oportunidad de aprendizaje en el momento mismo de ser evaluado. 
3. Para que el alumno interprete a los errores como fuente de aprendizaje, es conveniente que finalizado el examen y recogidas las pruebas, se brinde la respuesta correcta, para que los alumnos comenten y detecten sus errores, reflexionando sobre sus dificultades y aprendiendo en la instancia evaluadora.

4. Para evitar que a través de los ejercicios de aplicación sólo se incorporen hábitos no reflexivos, es conveniente plantear problemas en los que "el estudiante deba comenzar por reestructurarlo, a fin de darle una forma o un contexto similar a los que conoce en función de las ideas y métodos que espera le serán útiles para resolverlo, recién entonces usará correctamente estas ideas y métodos para encontrar la respuesta buscada. Para lograrlo deberá analizar, entre otras cosas, cuáles son las magnitudes pertinentes, si las conoce a todas o no, si el caso en cuestión se encuadra dentro de los límites de validez de la abstracción que va usar. Y finalmente deberá demostrar que es capaz de operar correctamente con los datos y abstracciones seleccionados" (Cudmani, L. C., Lozano, S. R. Lewin, A. F., 1981:274).

Por cierto que este análisis está limitado a un único instrumento: el examen final, el cual difícilmente pueda agotar todos los aspectos de una buena evaluación. Por ello, se considera que no debiera ser considerado como único instrumento para acreditar. Esta es una instancia mucho más abarcativa que la que se propone este trabajo.

\section{Conclusiones}

De acuerdo a los resultados obtenidos se confirmarían las hipótesis formuladas, en el sentido que la nueva estrategia metodológica, permite incorporar actividades evaluativas en el examen final que satisfacen, en buena medida, los principios establecidos por las normas NCTM (1989). Dadas las condiciones de infraestructura, el tiempo disponible para desarrollar la experiencia, y las reflexiones de distintos especialistas sobre las dificultades propias de la enseñanza de resolución de problemas, se considera que los instrumentos de evaluación empleados en el 2001, satisfacen los aspectos más importantes establecidos por el enfoque evaluativo elegido.

Esta conclusión ratificaría la información obtenida del análisis de otros instrumentos:

Los segundos parciales de 1999 y de 2001, en los que se pudo apreciar una mejora significativa en 2001 en el grado de corrección de la variable calidad de la asimilación, habiéndose también incorporado en esa instancia, actividades reflexivas sobre manejo de conceptos y de aplicación a las ciencias, actividades ausentes en los instrumentos empleados en 1999 (González de Galindo y Colombo de Cudmani, 2002 (a)), y

La encuesta realizada a los alumnos, en la que los mismos manifiestan una amplia adhesión a la nueva metodología, sugiriendo implementarla en la enseñanza de toda la asignatura (González de Galindo y Colombo de Cudmani, 2002 (b)). 
Estas conclusiones estimulan a extender esta estrategia metodológica a otros temas del currículo.

\section{Referencias bibliográficas}

Alonso, M., Gil, D. y Martínez Torregrosa, J. Los exámenes de Física en la enseñanza por transmisión y en la enseñanza por investigación. Enseñanza de las Ciencias, 10 (2), 127-138. 1992.

Arcavi, A....Y en Matemáticas, los que instruimos ¿qué construimos? Números. Revista de didáctica de las matemáticas. Vol. 38, pp. 39-56. 1999.

Ausubel, D. P., citado por Alonso, M., Gil, D. y Martínez Torregrosa, J. 1992. Los exámenes de Física en la enseñanza por transmisión y en la enseñanza por investigación. Enseñanza de las Ciencias, 10 (2), 127138. 1978.

Bruner, J. La educación, puerta de la cultura. Madrid: Visor. 1997.

Coll, C. y Martí, E. “Aprendizaje y desarrollo: la concepción genético-cognitiva del aprendizaje”. En Coll, C., Palacios, J. y Marchesi, A. Desarrollo psicológico y educación, II. Psicología de la educación, pp. 121-139. Madrid: Alianza Editorial. 1994.

Colombo de Cudmani, L., Pesa de Danón, M. y Salinas de Sandoval, J. La realimentación en la evaluación en un curso de Laboratorio de Física. Enseñanza de las Ciencias, 4 (2), pp. 122128. 1986.

Cudmani, L. C., Lozano, S. R. Lewin, A. F. El problema de aplicación como instrumento de aprendizaje operativo de física básica. Revista Brasileira de Física, Vol. 11, № 1, pp. 269-302. 1981.

Czar, M. y Pizarro de Raya, A. "Las corrientes psicológicas en el estudio del aprendizaje". En Czar, M. y Pizarro de Raya, A., Badfessi de Tapaltar, C. (Eds) Concepciones del aprendizaje y práctica docente. Módulo IV (pp. 3-64). Curso de Formación Pedagógica para Docentes Universitarios. Tucumán. Argentina: Secretaría de Planeamiento de la U.N.T. 1993.

Delgado Rubí, J. “Apuntes sobre la enseñanza problémica y la resolución de problemas". En Hernández Fernández, H., Delgado Rubí, J., Fernández de Alaíza, B., Valverde Ramírez, L. y Rodríguez Hung, T.: Cuestiones de didáctica de la matemática. Conceptos y procedimientos en la Educación Polimodal y Superior, pp. 89-98. Argentina: Homo Sapiens Ediciones. 2001.

Díaz Barriga, A. Docente y Programa. Lo Institucional y lo Didáctico. Argentina: Aique Grupo Editor S.A. 1990.

Díaz Barriga, F. y Hernández Rojas, G. Estrategias docentes para un aprendizaje significativo. (Una interpretación constructivista). México U.N.A.M.: Mc Graw Hill. 1997.

Gil, D., Carrrascosa J., Furió, C. y Martínez Torregrosa, J., citado por Alonso, M., Gil, D. y Martínez Torregrosa, J. 1992. Los exámenes de Física en la enseñanza por transmisión y en la enseñanza por investigación. Enseñanza de las Ciencias, 10 (2), 127138. 1991.

González de Galindo y Nieva de del Pino, M. “¿Es posible humanizar y democratizar la evaluación educativa en una facultad de ciencias?" Memoria 
del Congreso Internacional: University and Global Society, pp. 205-211. Logos Press. 2002.

González de Galindo, S. y Colombo de Cudmani, L. "Una propuesta innovadora para la enseñanza del cálculo en cursos superpoblados". Ponencia de la LI Reunión Anual de Comunicaciones Científicas y XXIV Reunión de Educación Matemática. 2001.

González de Galindo, S. y Colombo de Cudmani, L. "Evaluación de una experiencia para resignificar la clase magistral en la enseñanza del cálculo". Trabajo expuesto en la VI Reunión de Didáctica de la Matemática del Cono Sur, realizada en Buenos Aires. Argentina. 2002 (a).

González de Galindo, S. y Colombo de Cudmani, L. "Reflexiones sobre una experiencia didáctica en clases teóricas masivas de matemática: opiniones de los alumnos". Trabajo expuesto en el VI Simposio de Investigadores en Educación en Física realizado en Corrientes, Argentina. 2002 (b).

González de Galindo, S. y Villalonga de García, P. Una propuesta para el tratamiento metodológico de una clase de Matemática en una Facultad de ciencias. Revista IRICE, № 15, pp. 193-206. 2001.

González de Galindo, S., Villalonga de García, P., Nieva de del Pino, M., Chahar de Corrales, B., Holgado de Mejail. L. y Marcilla de Rulli, M. Una alternativa para enseñar Matemática en una Facultad de ciencias. Revista Científica de la Universidad de Blas Pascal, V 5, № 14, pp. 7-14. 2000.

Hernández Fernández, H.: "Vigotsky y la estructuración del conocimiento ma- temático. Experiencia cubana". En Hernández Fernández, H., Delgado Rubí, J., Fernández de Alaíza, B., Valverde Ramírez, L. y Rodríguez Hung, T.: Cuestiones de didáctica de la matemática. Conceptos y procedimientos en la Educación Polimodal y Superior. Argentina: Homo Sapiens Ediciones. 2001.

Moreira, M. "La teoría del desarrollo cognitivo de Piaget". En Moreira, M. (Ed.), Enfoques teóricos. Monografías sobre aprendizagem e encino. Brasil: Universidade Federal do Rio Grande do Sul. 1997.

NCTM. Estándares Curriculares y de Evaluación para la educación Matemática. Sevilla. España: Edición española. 1989.

Nunnally, J. M. Introducción a la medición psicológica. Buenos Aires. Argentina: Ed. Paidós. 1970.

Taylor, S.J. y Bogdan, R. Introducción a los métodos cualitativos de investigación. La búsqueda de significados. Buenos Aires: Editorial Paidós. 1987.

Villagra de Burgos, A. "La problemática de la evaluación", en Curso de formación pedagógica para docentes universitarios. Segunda parte. Módulo VI. Modelo Didáctico (pp. 77-110). Instituto Coordinador de Programas de Capacitación. Universidad Nacional de Tucumán. Argentina. 1993.

Villalonga de García, P. y Colombo de Cudmani, L. "La evaluación del aprendizaje en cursos básicos de Cálculo en una facultad de Ciencias". Memorias del IV Simposio de Educación Matemática. Chivilcoy. Buenos Aires. 2002. 